\title{
The Application of Random Walk Model to Enhance Utilization of Cloud based Services through Virtualization
}

\author{
Vaidehi M. \\ Dept. of Information Science and Engineering \\ Dayananda Sagar College of Engineering
}

\author{
T. R. Gopalakrishnan Nair, PhD \\ Dept. of Computer Science and Engineering \\ Raja Rajeshwari Group of Institutions
}

\begin{abstract}
Cloud computing has transformed the complete operation pattern of just not IT industries but also other sectors like Transportation, Metrological unit, Finances, education, agriculture, health care etc., The transformation is due to the enormous reliable services provided to these sectors at low cost. The Cloud model is able to do this because of the flexibility in providing services like the computing resource, storage or data managing and migration. These services are provided based on the user requirements, the user may opt for a public, private or a hybrid cloud.
\end{abstract}

Cloud based applications are fully deployed in cloud and all the supporting applications run in cloud. The performance of the computing model is influenced by the operation of the model. The computing model should always be available and reliable. Efficient operation depends on good scheduling and resource allocation techniques.

In this paper, a mathematical model to configure the required series of Virtual machines termed as Super Virtual machines (SVM) has been proposed. SVM is a combination of Virtual machines (VMs) which comprises of Processor, Storage space and Bandwidth required for computation. The research contribution shows an increase in availability and reliability of resources to requesting jobs.

\section{Keywords}

Super Virtual Machines, Availability, Reliability, Scheduling, Latency, Response time

\section{INTRODUCTION}

The Cloud technology has brought in a huge transformation in operation patterns of IT industries, financial, real estate business, and other major units where enormous data is generated and computed. This model has the capacity to provision resources dynamically to jobs submitted by its customers. The design of this model is such that the resources can scale up or scale down as per the job requirements. The customers expect the model to be reliable and secured. The applications that run in the cloud should assure the aforementioned customer expectations.

The datacenter manager should be capable of estimating the resource capacity required for computation or storage for a particular job to avoid resource mismanagement. Traditional datacenters provisioned more resources without the estimation of required resources for computation which affected the operational cost or the data centers had limited resources to cater to customer requirements leading to customer dissatisfaction.

Dynamic resource management and scheduling is one of the major responsibility of a Datacenter Manger to manage the effective utilization of Storage, CPUs or Database.

As this model is on-demand and pay-per-use, the costumers concern is high speed computation and less operational cost. They also demand innovative features which would enhance their business.

Virtualization is one of the important feature of Cloud which enables the jobs to utilize the allocated resources effectively.

Though the hardware and other applications required for computation are spread globally, due to Virtualization, the end users feel that the requested resources are exclusively allocated for them.

There are many service providers for example Amazon web Services which provides hardware and other applications to its users. The jobs submitted by these users can access the resources simultaneously,

This paper focuses on reliability and availability of the resources when requested by the jobs in computation queue. Here the proposed a Super Virtual Machine (SVM) comprises a chain of Virtual machines which can be built over any deployment model (Private, Public or Hybrid cloud). The objective of this model is to enhance the speed of operation, reliability and availability, and also reduce the operation cost.

The proposed model would be able to support homogeneous computation enabling agility and flexibility to users.

The organization of the paper is as follows, in section II the Background is presented, section III presents the Problem definition of the paper and proposed model, section IV depicts the Simulation setup and Performance Analysis. Section V depicts Conclusion and Section VI presents the References.

\section{BACKGROUND}

Researchers have investigated the problem of quick access of resources to enhance the performance, Rafael Xavier et.al, have proposed Elastic Media Resource provisioning algorithm for audio and video streams, the researchers say that the proposed algorithm has reduced total virtual machine cost by a maximum of 58\% when compared to other approaches [1].

Guangjie Han et al. have proposed Remain Utilization aware algorithm (RUA) for virtual machine placement and a PowerAware algorithm (PA) to find idle hosts to shut down for energy saving. The authors have combined the two algorithms for VM consolidations by using linear programming equations [2].W.M Ding et al, say that virtualization is being used by a growing number of organizations to reduce power consumptions. Virtualization enables Server consolidation, dynamic load balancing, disaster recovery, virtual desktops, and security issues. The authors say that virtualization provides high availability for critical applications and also 
streamline application deployment and migration through Cloud computing. They have proposed an architecture of Cloud computing based virtualization, and have evaluated the performance of the proposed system in terms of cost, energy utilization and time [3].

Xiong $\mathrm{Fu}$ and Chen Zhow say in their work that the placement of virtual machines in cloud data centers is an effective approach to enhance the resource utilization and reduce the energy consumption. The techniques Affinity based Virtual machine Placement algorithm) which explores the relationships for every two virtual machines based on the resource requirement. They say that the model evaluates the volatility of resource utilization after putting the virtual machines in the same host [4]

Shengmei et. al, in their research say that virtualization enhances the resource utilization by providing integrated operating environment for user and application based on accretion of heterogeneous and autonomous resources. The paper also presents that that virtualization enhances reliability, availability and reduces costs and provides flexibility [5]

Wei Zhe Zang et. al, in their work say that through virtualization multiple virtual machines can co-exist and operate on one physical memory. Here the VMs may contend for memory and the performance of the applications decline especially hose of memory intensive applications. The authors in their work say that they have optimized memory control techniques using balloon driver for consolidation. Their work is in three folds 1) Design and implementation of an automatic control systems for memory based on a Xen balloon driver.

2) They have designed an adaptive global scheduling algorithm to regulate memory 3) the optimized solution has been evaluated using test suites for 500 virtual machines [6]

\section{PROBLEM DEFINITION}

Efficient usage of resources in cloud environment is a challenge. There are various reasons for researchers to focus on utility of resources. As cloud environment is a pay-go model, the user should always look into the conservation and best utilization of resources.

In the cloud environment, generally the resources are not fully utilized either they are underutilized or over utilized affecting the efficiency of the Cloud environment.

Utilization of the resources through virtualization has few drawbacks.

A virtual environment would generally be preferred to reduce the investments made on hardware or software required for computing, but less effort has been put to see if the allocated resources are fully utilized.

Here in this paper the Random Walk technique has been applied. This approach allocates only the required size of the resources hence

\subsection{Proposed Algorithm}

The proposed algorithm is basically of type, demand driven. The virtual machine formation occurs based on the resource requests by the jobs in queue. The mapping of the resources through virtualization is to enable the system to perform efficiently by optimal utilization of the available resources.

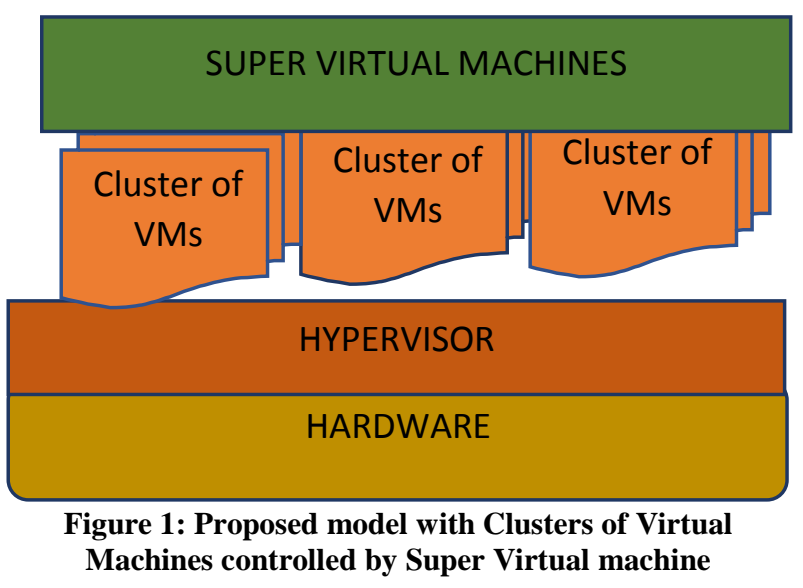

Figure 1, depicts the proposed model showing cluster of virtual machines controlled and coordinated by Super Virtual machines. These machines have an access to the hardware through a hypervisor. Here in our work we focus on virtualization of memory for computation of a submitted task. This approach is to enhance performance operation of the system through virtualization. Here Virtualization is a process of virtually sharing the required resources like processors, storage and bandwidth for computation of a task. The objective of our work is to map the three basic resources virtually and allocate the same to the requesting jobs in the queue to enhance the computation of the cloud model effectively and efficiently.

The Processor bank, Storage bank and the Bandwidth are mapped in such a way that the required mapping of these resources happen for optimal utilization of the available resources.

The figure 2 represents the three major resources mapped in accordance with the resources required for the computation.

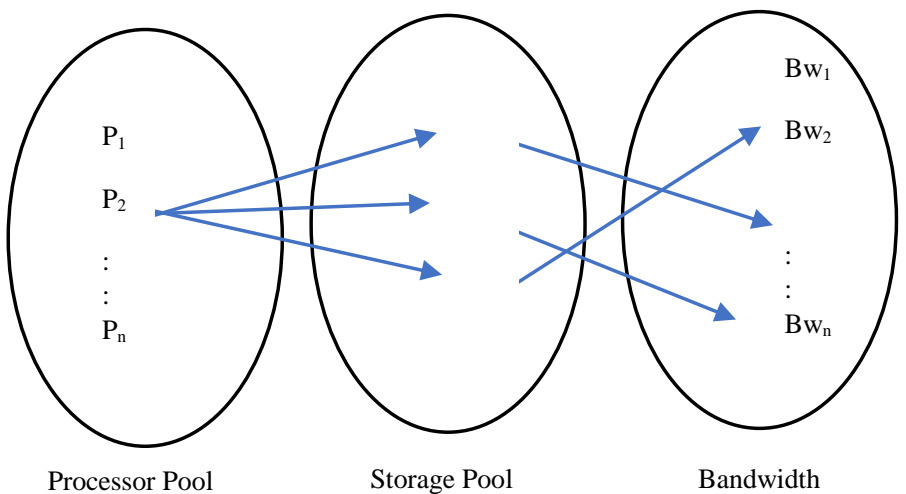

Figure 2: Mapping of Three major Resources

In this model we assume that there are ' $N$ ' Jobs $J_{1}, J_{2}, J_{3}, \ldots J_{n}$ in the queue are requesting for a Processor " $\mathrm{P}_{\mathrm{D}}$ ", Storage " $\mathrm{S}_{\mathrm{D}}$ " and Bandwidth " $\mathrm{B}_{\mathrm{D}}$ "

$\mathrm{P}_{\mathrm{D}}=$ Processor in demand

$\mathrm{S}_{\mathrm{D}}=$ Storage in demand

$\mathrm{B}_{\mathrm{D}}=$ Bandwidth in demand

The combination of the Processor, Storage and $b$

Bandwidth allocated for computation are defined as the Super Virtual Machine 
$\mathrm{VM}_{1}=\mathrm{P}_{\mathrm{D} 1}+\mathrm{S}_{\mathrm{D} 1+} \mathrm{B}_{\mathrm{D} 1}$

$\mathrm{VM}_{2}=\mathrm{P}_{\mathrm{D} 2}+\mathrm{S}_{\mathrm{D} 2}+\mathrm{B}_{\mathrm{D} 2}$

$\mathrm{VMn}=\mathrm{P}_{\mathrm{Dn}}+\mathrm{S}_{\mathrm{Dn}+} \mathrm{B}_{\mathrm{Dn}}$

Super Virtual Machine $=\mathrm{VM}_{1}+\mathrm{VM}_{2}+\ldots . \mathrm{VM}_{\mathrm{n}}$

From equation 1 " $\mathrm{P}_{\mathrm{D}}$ " the total processor demand $\mathrm{P}_{\mathrm{D}}$ can be realized through integrating of several processors having interconnectivity and required fragments of storage. The fragments are identified from the matrix $\mathrm{P}_{11} \ldots . \mathrm{P}_{\mathrm{mn}}$ as shown in figure 3 .

$$
\left[\begin{array}{ccc}
\mathrm{P} 11 & \mathrm{P} 12 \mathrm{P} 13 \ldots \ldots \ldots \ldots \ldots . \mathrm{P} 1 \mathrm{~m} \\
\mathrm{P} 21 & \mathrm{P} 22 \mathrm{P} 13 \ldots \ldots \ldots \ldots \ldots \ldots . \mathrm{P} 2 \mathrm{~m} \\
\mathrm{P} 31 & \mathrm{P} 32 \mathrm{P} 33 \ldots \ldots \ldots \ldots \ldots \ldots \mathrm{P} 3 \mathrm{~m} \\
& ; \\
& & \\
\mathrm{Pm} 1 & \mathrm{Pm} 2 & \mathrm{Pm} 3 \ldots \ldots \ldots \ldots . . \mathrm{Pmn}
\end{array}\right]
$$

Figure 3 Fragments of Processors

So in the matrix all processors having processor time will not be suitable for making $\mathrm{P}_{\mathrm{D}}$ because of non-matching storage and non-matching bandwidth between them.

So, $\mathrm{P}_{\mathrm{D}}=$ optimality of a finite set is taken from $\mathrm{P}_{\mathrm{nm}}$ matrix, $\mathrm{S}_{\mathrm{nm}}$ matrix and $\mathrm{B}_{\mathrm{nm}}$ matrix. After selecting the appropriate processor map from the PMS bay it looks like

$\mathrm{P}_{\mathrm{D}}=\sum \operatorname{Pnm}\left(\sum \mathrm{S}_{\mathrm{nm}}\left(\sum \mathrm{B}_{\mathrm{nm}}\right)\right)$

Since this is an optimality problem first hand implementation can be done through a minimization of the available process capability function.

From the study carried out on the existing systems it was observed that the resources allocated were not completely utilized by the requested jobs while computation or the jobs had a smaller amount than the required resources for computation. To overcome these drawbacks, we have applied the Random Walk model to the proposed mapping technique as shown in figure 2 .

\subsection{Random Walk Model}

\section{Resource formation through Virtualization Model.}

Random Walk is a stochastic process formed by continuous summation of independent identical distributed random variables.

With reference to the figure 2 , the resources of various size are allocated to the requesting jobs in fixed probability as shown in figure 3

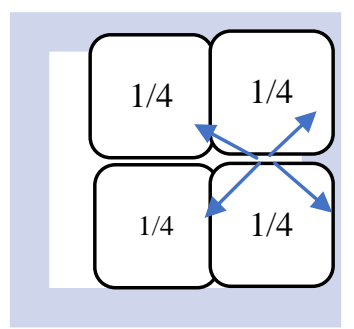

Figure 4 Probability of resource allocation

Figure 3 is sample of cells called as Super Virtual Machines
(SVM) comprising of the available resources mapped as shown in figure 2 . Resource grouping has been taken for resources allotment to requesting jobs.

Each cell is a combination of the three major resources (Processors, Storage and Bandwidth).

Each cell varies with different capacities. Based on the request, a minimum one of the resources combination represented by each cell will be allocated. The selection on cells depends on the Random walk model.

The application of the Random Walk is explained with the help of the figure 4 .

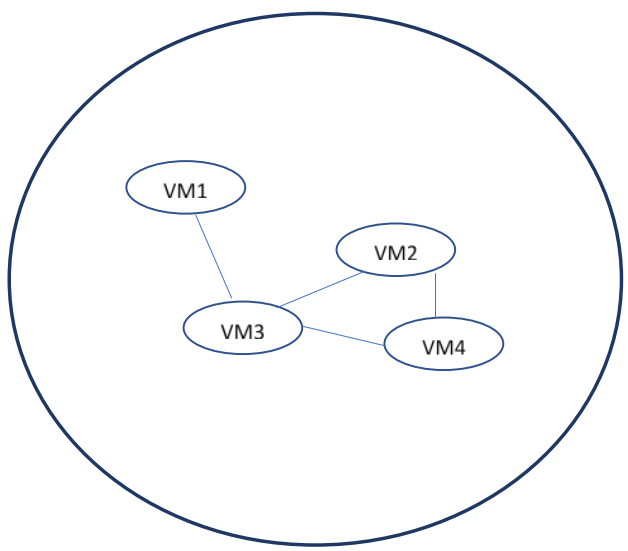

Figure 5 Selection of Virtual Machines from a pool of Virtual machines based on undirected Random Walk Random Walk by SVM

\subsection{Formation of Super Virtual Machine} (SVM)

$\mathrm{SVM}_{\mathrm{ij}}^{\alpha}=\frac{\alpha \mathrm{i} \mathrm{Zij}}{\sum \mathrm{k} \alpha \mathrm{kkj}}$

$\mathrm{Zij}$ : represents the weight of the Super Virtual machine

It selects current machine ' $\mathrm{j}$ ' to ' $\mathrm{i}$.' the next machine

The probability of selecting VM from $j$ to $i$ is given by

$\mathrm{P}^{\alpha}{ }_{\text {svm ij }}=\alpha i Z i$

\section{SIMULATION SET UP}

The Random walk algorithm was configured with the Cloud analyst tool. The tool supported with creation of data centers. These centers are provided with variable resources like, Storage, Bandwidth and Processors. Based on the job size, these resources for clusters of SVMs. The formation happens through the application Of Random walk algorithm.

Table 1: Configuration of Hardware Resource

\begin{tabular}{|c|c|c|c|}
\hline $\begin{array}{c}\text { Data } \\
\text { Center }\end{array}$ & Region & Architecture & VMM \\
\hline DC1 & 1 & X86 & XEN \\
\hline DC2 & 1 & X86 & XEN \\
\hline DC3 & 1 & X86 & XEN \\
\hline DC4 & 2 & X86 & XEN \\
\hline DC5 & 2 & X86 & XEN \\
\hline
\end{tabular}




\section{RESULT ANALYSIS}

From the simulations it is observed that the resources are quickly available to the requesting jobs and hence has improved the response time of the system.

Few samples of response time are shown. The SVMs have enhanced the system performance through improved response time

Figure 6 shows the comparison of Availability of the resources at time' $t$ ' using SVMs and VMs.

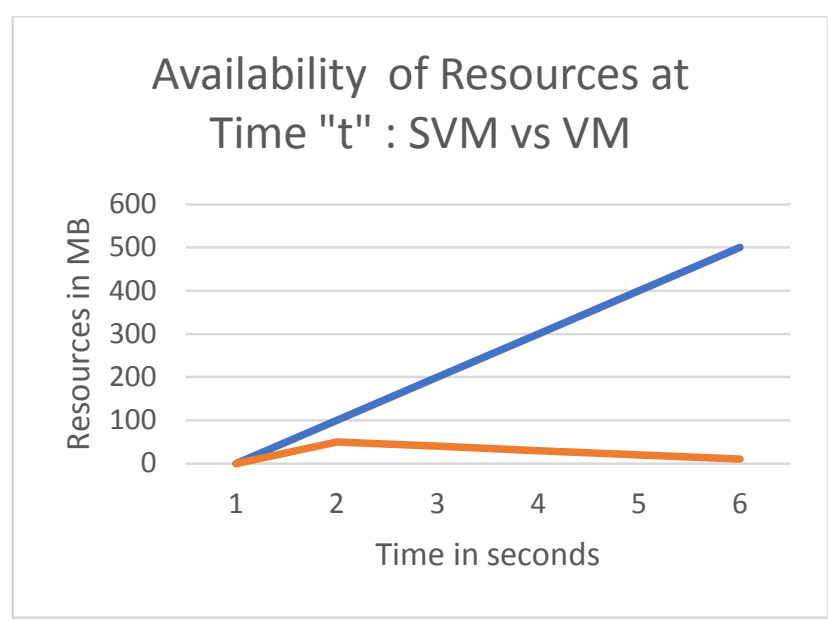

Figure 6: Availability of Resources using SVMs and VMs

\section{CONCLUSION}

As application of the Cloud model is exponentially increasing in the current scenario, customer satisfaction and retention is one of the essential requirement for the computing model to be a successful one.

This can be accomplished through better performance of the system. Performance depends on factors like availability of resources, quick response time, and improved throughput. To achieve quick availability of resources, here in our work we proposed the application of SVMs, and compared it with the application of Virtual machines to the same set of jobs in queue. The results showed improved performance in terms of the availability of resources which were chosen by the application of Random Walk model. It is also observed that the services provided is efficiently utilized by the jobs through SVMs formation.

\section{REFERENCES}

[1] Jose Rafel Xavier Dos Santos, Hendrik Moens, Bruno Vokkaert, Flip De Truck, "Design and evaluation of elastic media resource allocation algorithms using CloudSim extensions", International Conference on Network and Service Management 2015, pp 318-326

[2] Guangjie Han, Wehui Que, Gangyong Jia, and Lie Shu, An Efficient Virtual Machine Consolidation Scheme for Multimedia Cloud Computing, Sensors, 2016, 16,246.DOI:10.3390/S16020246, mdp.com/journal/sensors

[3] W. M. Ding et al., "Research on the Virtualization Technology in Cloud Computing Environment", International Journal of Engineering Research in Africa, Vol. 21, pp. 191-196, 2016

[4] Xiong Fu and Chen Zhou, "Predicted Affinity Based Virtual Machine Placement in Cloud Computing Environments", IEEE Transactions, on Cloud Computing, Vol 13, No.9 September 2014, DOI: 10,1109/TCC.2017.2737624,IEEE

[5] Shengmei Luo, Zhaoji Lin, Xiaohua Chen, Virtualization Security for Cloud Computing Service", International Conference on Cloud Service Computing 2011

[6] Wei-Zhe Zhang, Hu-Cheng Xie, and Ching-Hsien Hsu, Automatic Memory Control of Multiple Virtual Machines on a Consolidated Server, his article has been accepted for publication in a future issue of this journal, but has not been fully edited. Content may change prior to final publication. Citation information: DOI 10.1109/TCC.2014.2378794, IEEE Transactions on Cloud Computing

[7] Anton Beloglazov and Rajkumar Buyya "Energy Efficient Resource Management in Virtualized Cloud Data Centers"2010 10th IEEE/ACM International Conference on Cluster, Cloud and Grid Computing.

[8] Wenxin Li, Heng Qi, Keqiu Li, Ivan Stojmenovic, and Julong Lan, "Joint Optimization of Bandwidth for Provider and Delay for User in Software Defined Data Centers", IEEE TRANSACTIONS ON CLOUD COMPUTING, VOL. 5, NO. 2, APRIL-JUNE 2017

[9] Meryeme AouanE, Hanan El Bakkali, "Virtualization in Cloud Computing: NoHype vs HyperWall New approach", 2nd International Conference on Electrical and Information Technologies ICEIT'2016

[10] Nancy Jain, Sakshi Choudhary, "Overview of Virtulization in Cloud Computing", Symposium on Colossal Data Analysis and Networking (CDAN) 2016

[11] Suneeta Mohanty, Prasant Kumar Pattnaik, G. B. Mund, “ Privacy Preserving Auction Based Virtual Machine Instances Allocation Scheme for Cloud Computing Environment', International Journal of Electrical and Computer Engineering (IJECE) Vol. 7, No. 5, October 2017, pp. 2645 2650 ISSN: 2088-8708, DOI: 10.11591/ijece.v7i5.pp2645-2650

[12] .Kuo-Hsing Chiang and Nirmala Shenoy A 2-D Random-Walk Mobility Model for Location-anagement Studies in Wireless Networks", IEEE TRANSACTIONS ON VEHICULAR TECHNOLOGY, VOL. 53, NO. 2, MARCH 2004

\section{APPEDIX}

Samples of SimulationS 


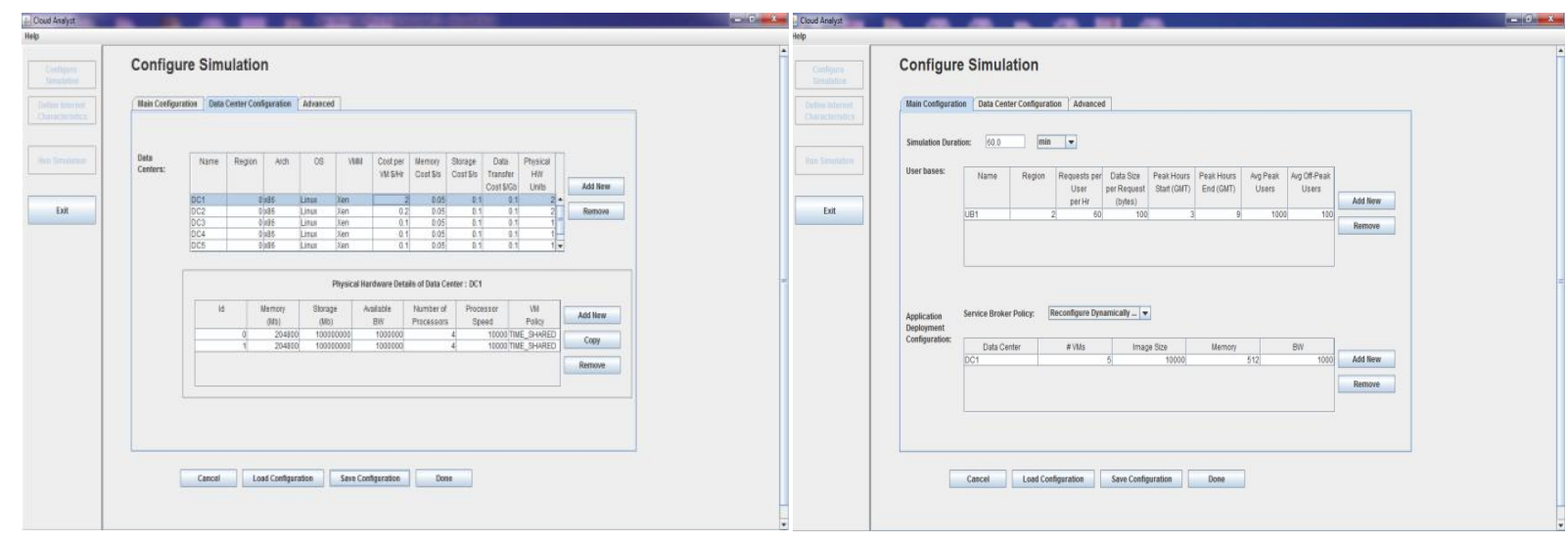

Results of the Simulation Completed at: 13/12/2018 13:11:02

Overall Response Time Summary

$\begin{array}{llll} & \text { Avg (ms) } & \text { Min (ms) } & \text { Max (ms) } \\ \text { Overall response time: } & 300.31 & 229.60 & 373.67 \\ \text { Data Center processing time: } & 0.51 & 0.02 & 9.50\end{array}$

Response Time by Region

\begin{tabular}{|l|l|l|l|}
\hline Userbase & Avg (ms) & Min (ms) & Max (ms) \\
\hline UB1 & 290.82 & 246.27 & 363.51 \\
\hline UB2 & 301.09 & 241.63 & 370.66 \\
\hline UB3 & 299.70 & 241.71 & 369.26 \\
\hline UB4 & 300.65 & 234.39 & 373.67 \\
\hline UB5 & 300.26 & 229.60 & 366.37 \\
\hline
\end{tabular}

User Base Hourly Response Times

UB1

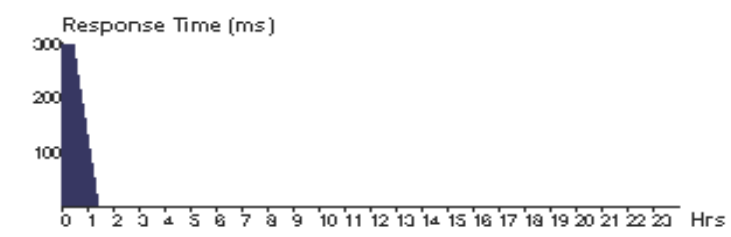

UB2

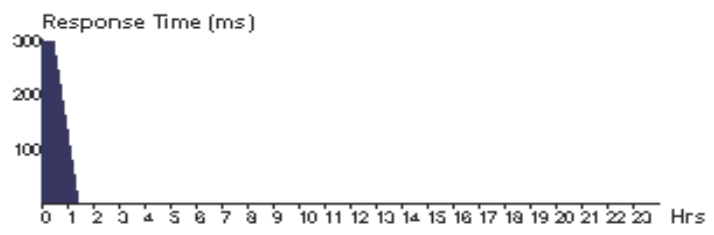

UB3

UB4 
Results of the Simulation Completed at: 13/12/2018 13:43:01

Overall Response Time Summary

Overall response time:

Data Center processing time:
Avg (ms)

359.45

0.89
Min (ms)

233.16

0.03
Max (ms)

636.12

2.99

Response Time by Region

\begin{tabular}{|l|l|l|l|}
\hline Userbase & Avg (ms) & Min (ms) & Max (ms) \\
\hline UB1 & 302.95 & 233.16 & 381.38 \\
\hline UB2 & 457.92 & 338.53 & 636.12 \\
\hline UB3 & 315.48 & 251.44 & 405.87 \\
\hline
\end{tabular}

User Base Hourly Response Times

UB1

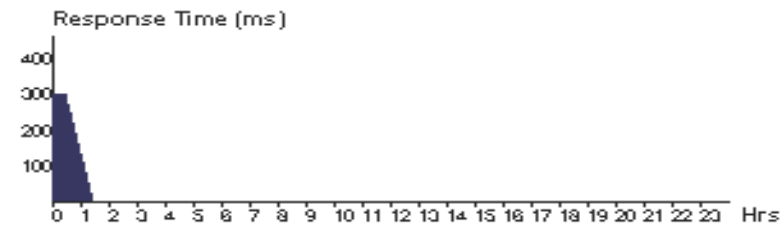

UB2

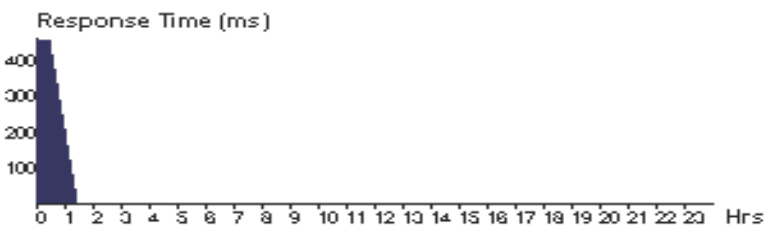

UB3

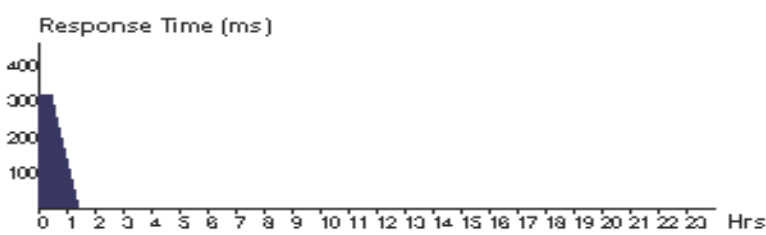

